\title{
On the Dynamics of
} Communication and Cooperation in

\section{Artificial Societies}

\section{A.E. Eiben M.C. Schut N. Vink}

Department of Computer Science, Artificial Intelligence Section, Vrije Universiteit, Amsterdam, The Netherlands

\section{Key Words}

Communication $\cdot$ Cooperation $\cdot$ Artificial societies $\cdot$ Evolutionary learning

\begin{abstract}
This paper compares two fundamental building blocks of complex interactionbased systems: communication and cooperation. We investigate the effectiveness of communication in an environment where the need for cooperation is scalable as well as the available resources. Several aspects of communication are considered: firstly, we compare a centralized with a decentralized communication protocol; secondly, we compare a population that always communicates with one where the entities can (evolutionary) learn to communicate. This work is part of a larger project whose main goal is to investigate the emergence of cooperation and communication in response of (scalable) environmental challenges. Our application context is an artificial society, i.e., a simulation of a societal system that was inspired by the classical SUGARSCAPE that embodies a bottom-up approach to investigate complex effects that do not necessarily have complex causes.
\end{abstract}

Copyright $\odot 2005$ S. Karger AG, Basel

\section{Fax +4161306 1234 E-Mail karger@karger.ch www.karger.com}

KARGER (c) 2005 S. Karger AG, Basel 1424-8492/05/0024-0152 $\$ 22.00 / 0$

Accessible online at: www.karger.com/cpu
M.C. Schut, Department of Computer Science, Artificial Intelligence Section, Vrije Universiteit, De Boelelaan 1081, NL-1081 HV Amsterdam (The Netherlands) Tel. +31 20598 7668, Fax +31 20598 7653, E-Mail gusz,schutg@cs.vu.nl or nvink@illyan.nl

\section{Simplexus}

Communication is a fundamental resource for building cooperation among 'agents' of any kind - from bacteria or people to nations, or artificially intelligent robots. The more easily agents can communicate and share information, the better chance they can learn to coordinate their actions in a beneficial way. It is no accident that bacteria, for example, lead intensely social lives and have myriad chemical mechanisms for communication, which enable colonies to carry out tasks far more delicate than any bacterium could manage on its own, from hunting large prey to devising collective strategies for defeating host immune responses.

In this paper, Schut and colleagues begin to explore the nature of the relationship between communication and cooperation by examining how two specific schemes for communication fare in enabling cooperation among a group of agents. In simulations using a generic model of interacting agents who must cooperate in order to survive, they demonstrate that changes in the mechanism of communication can strongly influence the fate of an agent population, especially under harsh environmental conditions. The present study is rather preliminary, yet Schut and colleagues argue that further studies along these lines should help to identify key mechanisms by which communication can help agents to achieve important collective goals, either in natural or artificial settings. This kind of knowledge may be especially important for engineering desirable kinds of self-organized cooperation in the Internet or in other technological applications, such as the management of swarms of robots.

From microbes to business firms, the agents making up complex social or biological systems have intentions and aims that reflect their individual needs - to eat and reproduce, for example, or to make sufficient profits. In general, communication enhances the possibility that many agents can coordinate their activities to 


\section{Introduction}

The building blocks of a complex system with many active entities are communication and cooperation. Communication is used for information exchange between the entities and cooperation is necessary if the entities want to achieve goals that are beyond their own reach. If we are to design complex artificial systems, we have to know the effects of deciding for available ways to communicate with respect to a given problem environment. This paper presents a first step towards such design questions.

We investigate the communication and cooperation properties of a complex system with many interacting entities among three different dimensions.

- Firstly, we vary the available resources and the cooperation threshold; the resources are needed by the agents to survive, the cooperation threshold determines how strong the pressure is on the agents to cooperate.

- Secondly, we compare (1) a centralized communication protocol - where individuals multicast messages that can be received by any individual - and (2) a decentralized communication protocol where information is transferred directly between agents without a third party (messageboard or alike).

- Thirdly, we parameterize the communication protocols (e.g., probability that an agent talks or listens to other agents) and we empirically compare the implications of (1) fixing these parameters and (2) letting the agent learn these parameters themselves.

Our agents are equipped with a hardwired mechanism for communication, and learn (by evolution) to use this mechanism. Our notion of cooperation is rooted in the environment. It is interesting to mention that our approach is complementary to some of the classics. Namely, we study the emergence of communication under fixed properties of cooperation (hard-coding its mechanics), while many studies focus on the emergence of cooperation under fixed properties of communication (see for instance Axelrod [2], who assumes there is none).

Our experiments are conducted in a straightforward artificial society. This society consists of a collective of agents that lives off harvesting sugar resources in the environment. In some situations, agents may be forced to harvest sugar together with other agents. Each agent is able to communicate information about the amount of sugar at its location and may also receive such information from other agents.

Our main research objective can now be specified as follows: to study the development of the agents' communication attitude (talk/listen gene distributions) and cooperative behaviour (eating together) under varying levels of cooperation pressure (maximum amount of sugar they can eat alone).

This paper is organized as follows. In section 2 we explain the concept of an artificial society, the communication protocols that we researched and some earlier work that we did on this topic. Section 3 describes the system that we designed for carrying out the experiments. Section 4 contains the setup of the experiments and presents the obtained results. Section 5 analyses the results. We conclude and present future work in section 6 .

\section{Background}

\subsection{Artificial Societies}

Our research can be positioned in a broader context, that of artificial societies. We let artificial societies be agent-based models of social processes [11]. This definition brings with it some notion of agents (the 'people' of the artificial society), simulation (models are computationally executed to explore societal phenomena) and social structures (the macroscopic behaviour of a group of interacting individuals).

Epstein and Axtell [11] let an artificial society consist of (1) agents, (2) an envi- achieve what none could achieve alone, so that each draws advantages from membership in a 'community'. Bacteria collaborate to produce 'biofilms' that give them collective resistance to anti-bacterial agents, or modern multinational corporations that pool their distinct areas of expertise for strategic purposes. Of course, the precise nature of communication among such agents - the mechanism by which it takes place - should influence the level of cooperation, and therefore a population's ability to survive in more or less difficult environments.

To explore this influence in detail, Schut and colleagues carried out a series of computer simulations of agents living on a twodimensional grid of squares. In their artificial world, the agents face a very basic challenge: to harvest enough 'sugar' (a source of energy) to stay alive and reproduce. At any moment, an agent can either harvest sugar - if it finds plenty at its present location - or try to move toward other sources elsewhere. The authors, as the world's designers, can alter how much sugar there is in the environment, making it more or less challenging.

Crucially, the agents have two key limitations. On their own, they're able to harvest only a limited amount of sugar per unit time. Also, they can only see other sources of sugar that are close enough to their present location (their vision is limited). These limitations make cooperation important for the agents' survival, and implicitly make communication matter, in two particular ways. The authors allow agents to share information on food sources they have seen. By 'listening' to others, an agent can learn the whereabouts of promising sources of sugar, and thereby take steps to move toward them. Meanwhile, by 'talking' to others, agents in the midst of plenty can hope to recruit help that will increase their own harvest. The effects of such communication will in general depend on the mechanisms through which it takes place, and exploring this ef- 
ronment or space, and (3) rules. An agent then has internal states and behavioural rules, which each can be fixed or flexible. Interactions and changes of internal states depend on rules of behaviour for the agents and the space. Environments can be abstractly defined (e.g., a communication network) or more resemble our own natural environment (e.g., a lattice of resourcebearing sites). The environment is a medium separate from agents, on which the agents operate and with which they interact. Rules can be defined to describe the behaviour of agents and the environment on different interaction levels, i.e., agent-environment (e.g., agents looking for and consuming food), environment-environment (e.g., growing resources), and agent-agent (e.g., combat and trade).

\subsection{Communication}

As mentioned, our research compares a centralized with a dentralized communication protocol.

\section{Centralized Communication}

In the centralized approach, communication between agents is supported by a centralized component; this is a component that is accessible by all agents, e.g., communication through a messageboard. A messageboard enables the agents to communicate by facilitating the storage and retrieval of communicated information. All agents wanting to communicate can access the board to post a message to it, or read messages posted on it.

\section{Decentralized Communication}

In the decentralized approach, there is no central support for communication. Agents that wish to communicate to other agents need to manually find agents to communicate to and exchange the information with them. Many decentralized communication protocols (e.g., gossip, epidemic-based) have been proposed and researched recently. We have implemented the newscast model [12] in the experi- ments described below that we explain in more detail.

\section{Newscast Communication}

The newscast computing model is a fully distributed information propagation protocol for large-scale peer-to-peer computing $[12,14]$. The main idea of newscast is that each agent maintains a cache of information items holding the information for and from the agent; the cache also contains the names of all agents that are 'friends' with the agent. The cache of names, i.e., IDs and addresses, is used each time a communication is initiated by the agent. Each agent can listen and receive the messages from other agents that have it in their cache. At fixed time intervals, the agent updates the information in its cache and the list of names.

The used metaphore for illustrating the newscast model is the concept of a news agency. This agency regularly asks all agents for news. Additionally, the agency provides each agent with news about the other agents in the society.

Each agent has a correspondent module that maintains a cache of $c>0$ news items, where $c$ is fixed. A news item contains a timestamp, the agent ID and the message itself (location + sugar amount). Agents regularly exchange their caches by following this procedure (where the local agent is the agent who initiates an exchange with a peer agent):

1 Request a fresh news item from the local agent and merge the item into the cache.

2 Randomly select a peer correspondent by considering its ID as found in the cache. 3 Send and receive each other's caches. Merge received items into the local cache.

4 Since the cache now contains $2 c+1$ cache items, the oldest ones are thrown away to keep the $c$ freshest ones (breaking ties randomly).

\subsection{Scalable Environments}

As mentioned, the work presented here is part of a larger research project investi- fect is the main point of the paper. The authors explore two possibilities.

Communication might take place through a centralized 'messageboard' to which all agents can post information (for example, giving the location and amount of a source of sugar they have seen). In this case, information offered by one agent may be picked up by any agent in the population. Alternatively, communication might be decentralized, with agents communicating only with specific others to whom they are linked in a 'peer-to-peer' network of some kind. In effect, this network works a little like a messageboard, yet one that is restricted to a smaller subgroup of the whole population.

The authors' figure 1 depicts the basic biology of a virtual world in which they tested the consequences of these different communications protocols. This flow chart captures the logic by which agents' actions follow from the situations in which they find themselves. Of particular importance, agents in some of the simulations did not have to make use of the communication possibilities at all times, but could merely have a propensity to do so. Moreover, such propensities were subject to evolution by natural selection. Agents were given a rudimentary form of genetic inheritance, with the population reproducing through pairwise mating, whenever two agents of opposite sex land on the same square. Those able to harvest more sugar tend to contribute more offspring to later generations. To make the evolution of real novelty possible, the authors also include rare mutations, which can lead to agents with entirely new phenotypes (being more or less likely to listen than their parents, for example).

In this context, the authors ran simulations while varying four key parameters:

MaxSugarHarvest-a parameter setting how much sugar an individual agent can harvest before it needs the help of others;

MaxSugarSize - a parameter that controls the overall level of sugar resources, 
gating the (communication) responses of an artificial society to scalable environmental challenges (here: cooperation). As such, this work extends earlier work [9] where we also compared the centralized messageboard protocol with the decentralized newscast protocol, but where we fixed the cooperation threshold and available resources on single values. Other work [5] investigated the message removal methods for the messageboard protocol. We investigated the following settings: (1) removal after fixed number of iterations; (2) removal after the message has been listened to, and (3) removal after cooperation took place resulting from the message. No significant differences between methods 1 and 2 were found, whereas method 3 consistently resulted in early extinction of the society. In the work reported here, we used removal method 2.

In related work [4] we investigated the agent's learning capabilities to develop physical and mental properties. We researched both lifetime and evolutionary learning. The results indicate that the evolutionary approach is able to sustain larger and more stable agent populations as well as maintain a higher degree of individual success compared to the lifetime learning approach. Furthermore, quite unexpectedly, the method used for mental development has a strong effect on the development of the physical features within the very same environment: the individuals' bodies evolve to completely different segments of the physical feature space under the two regimes.

In a previous study [8] we extended (1) the environment to include a heterogeneous set of resources, and (2) the agent's reproduction mechanism as to where and when reproduction took place. For the first extension, the environment presented here knows only one type of resource (defined in terms of the benefit to the agent utilizing this resource), whereas in the previous study [8] we researched three different types of resources. For the sec- ond extension, agents could reproduce (1) only with their neighbours or with anyone in the environment and (2) during their lifetime or only at the end of their lifetime. The results show that utilizing reproduction at the end of an agent's lifetime and local reproduction (only with neighbours) afforded the agent collective a significantly higher level of performance in its cooperative task.

Finally, in another study [9] we also present a preliminary theoretical model on the relationship between the decay of value of information that agents act upon and the rate at which agents exchange information with each other. The environments that we investigate turn out to have a very rapid decay of information value: a communicated message may long have lost its value once it reaches its listeners. This puts the results found in these environments in the following perspective: the used communication protocol must be able to remove outdated information fast enough. We later indeed find that the newscast protocol (due to its decentralized nature) is not able to remove outdated information fast enough. Because our decentralized communication protocol (messageboard) removes information once it has been listened to, this gives it an advantage in these environments already from the outset.

\section{System Description}

The system in which we conduct our experiments consists of a simulated environment that represents an artificial society (called VUSCAPE) and a set of agents that populates this society.

\subsection{JAWAS}

The JAWAS ${ }^{1}$ simulation platform is comparable with existing social simulation software such as Ascape [13], Repast [6] and Swarm [7]. All settings in JAWAS

${ }^{1}$ JAWAS, Java Artificial Worlds and Agent Societies, can be down loaded from http://www.cs.vu.nl/ci/eci/. making it easier (plentiful sugar) or harder (scarce sugar) for agents to survive;

Communication protocol - a binary parameter determining the type of communications used by the agents, either centralized ('messageboard') or decentralized ('newscast');

Communication flexibility - a binary parameter determining if the agents always make full use of their communications possibilities (that is, they always talk about sugar sources and listen to what others have to say), or if this behaviour is instead subject to evolution, each agent having a propensity for talking and listening which can pass down to offspring.

The results of these experiments appear in figures 2 through 4 , which display how different communication protocols and listening/talking behaviour influenced agents' ability to survive in differing environments, and, in some cases, how the agents'listening/talking behaviour evolved to produce better communications under the prevailing conditions.

In figure 2 , every point in the two-dimensional space corresponds to particular values of MaxSugarHarvest and MaxSugarSize, which set the harshness of the environment and the threshold at which individuals have to cooperate to harvest sugar. Environments get harsher moving downward, and cooperation becomes more important toward the left. Each curve, for a particular communications strategy, shows a cutoff below which all populations started under these conditions ultimately died out. The uppermost curve is the baseline performance for no communications at all; populations die out very easily, and do so more readily when cooperation is more important (again, to the left). They survive quite well when cooperation is unimportant (far right), in which case the kind of communication also does not matter.

The series of curves moving downward from here then shows the consequences of different kinds of communication. In gen- 
can be specified either in configuration files or via command line arguments. This enables the user to automate experiments, which substantially speeds up the time needed for, for example, investigating effects of varying experimental parameters (often requiring a large number of runs). Data are automatically saved at specified locations, enabling detailed experimental logging. Thorough statistically based experimental research on complex systems that depend on numerous parameters requires a large number of runs. Facilitating this is one of the main design objectives of JAWAS. It is very easy to add, replace or delete code when changes or extensions to the model need to be implemented. No direct connection is necessary between the program and the graphical user interface. Initial exploration can use the graphical user interface, and for automated experimentation, the system can be run solely with configuration files or from the command line.

\subsection{Simulated Environment}

The simulated environment is an artificial society called VUSCAPE [5], which is based on SUGARSCAPE [11]. This artificial society concerns a two-dimensional grid, wrapped around the edges, where each position corresponds with an area which can contain multiple agents and some amount of sugar. Agents move through the world by vertically or horizontally jumping to another location (see moving in SUGARSCAPE). The agents live off the sugar, determining their level of fitness; if an agent's fitness reaches zero, it dies. The major differences between VUSCAPE and SUGARSCAPE concern the implementations of cooperation, communication, explorative behaviour, increased grid-point inhabitance, randomized sugar distribution, and randomized age initialization. We investigated the effects of these differences experimentally in an earlier study [5].

\section{Cooperation}

Each agent can only harvest a maximum amount of sugar on its own. This amount is set by the maximum sugar harvest (MSH) parameter. If an agent is at a location at which the amount of sugar is over this threshold, it needs other agents to harvest the sugar. If there are more agents at such a location, these agents harvest the sugar together and the sugar is evenly distributed over these agents. In the experiments described below, the cooperation threshold is the same for all agents.

In addition to the MSH parameter, we scale the necessity to cooperate by varying the number of available resources in the environment, called the maximum sugar size (MSS) in VUSCAPE. Based on the settings of MSH (implements the earlier mentioned cooperation threshold) and MSS (implements the earlier mentioned available resources), we can create easier and more difficult environments for the agents to survive.

\subsection{Agents}

Our agents were based on the classical SUGARSCAPE agent design: prominent features include metabolism, gender, child bearing, death, vision, allow sex and replacement. An agent was able to detect agents and resources for a number of grid cells determined by its vision parameter. It was able to move for a number of grid cells determined by its move parameter.

The control loop of the agent is shown in figure 1:

- An agent gathers information about the distribution of sugar in the world. This is done by means of listening (to other agents) and looking (at the directly surrounding locations and the current location). Upon completion of this stage, the agent has at its disposal an array of locations and amounts of sugar on these locations.

- Based on this array, the agent makes a decision about its next action. In particu- eral, the conclusion is that the centralized communications mechanism ('messageboard') gives better performance than the decentralized mechanism ('newscast'). This remains true whether the agents always listen and talk, or if they can evolve. The local newscast strategy works better if the agents can evolve, as one might expect. A rather surprising finding, however, is that the centralized messageboard mechanism actually performs worse when the agents can evolve than when they cannot.

As the authors conclude, communication does help agents, especially when their need to cooperate is urgent. When cooperation is unimportant (in the right of the diagram), the kind of communication does not matter, as reflected in the collapse of all curves onto just one line.

The second outcome of the experiments, depicted in figures 3 and 4, shows how the listening/talking behaviour of the agents evolves to respond to the prevailing conditions. Figure 3 shows results for the centralized ('messageboard') mechanism of communications. In general, the figures show that as conditions get worse (moving left in the diagrams, toward lower MaxSugarSize) surviving agents have evolved a high tendency to talk. In the surviving populations, the agents have also evolved to listen with probability approaching one. These tendencies are not so clear in figure $3 c$, however, where the need for cooperation is very low. The outcomes in figure 4 , for the decentralized ('newscast') style of communication, show no consistent evolution of the talk levels under different needs to cooperation.

These results are not entirely surprising. One would intuitively expect that the need for communications should show itself more readily under difficult conditions that demand greater cooperation, and moreover that centralized communications, because they connect more of the population, should generally outperform more local connections. Of course, in real settings in biology or modern technology, 


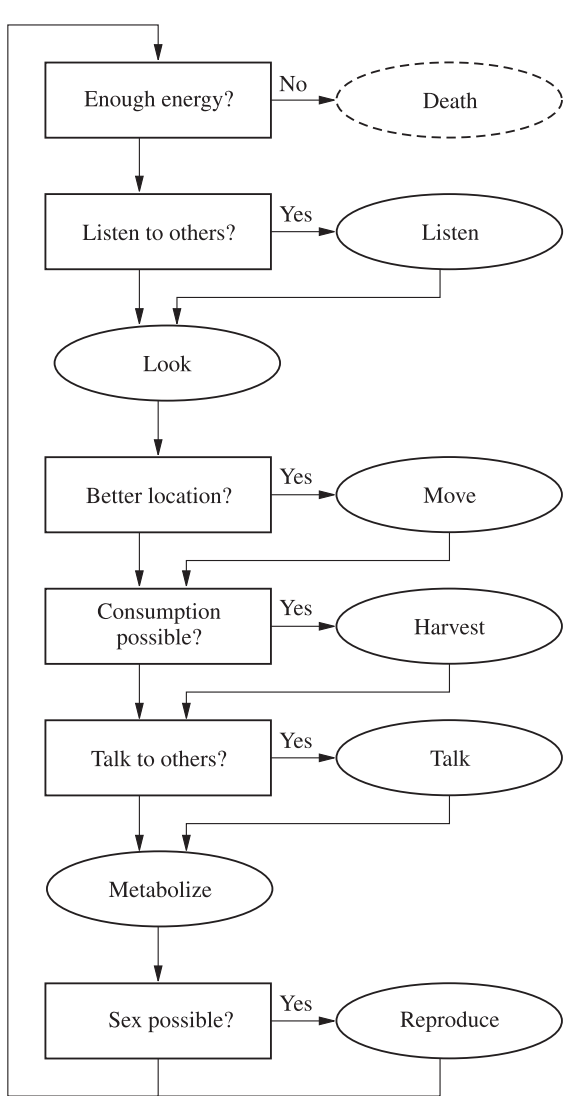

Fig. 1. The agent control loop.

lar, it chooses a location and moves to this location. This location chosen is always the one containing the largest amount of sugar. If there are more than one such location, the agent chooses a random one.

- Having arrived at the sugar, this sugar is harvested in case the amount is under the cooperation threshold. If the amount is above the cooperation threshold, the agent cooperates immediately if there are more agents at the location. Otherwise, it multicasts (with some probability) to other agents that it needs help.

- If possible, the agent reproduces and generates offspring. For this, it is (at least) necessary that there is another agent of the opposite sex at the location.

\section{Communication}

Agents are endowed with talk and listen capabilities. The talk feature determines whether the agent performs a communicative action itself, namely informing other agents of: (1) the amount of sugar that is on its location, and (2) the coordinates of its location. The listen feature is used in the observation and decision making processes of the agent. By listening, the agent receives information from other agents about amounts of sugar at the locations of those agents.

The listen and talk genes express probabilities and are formally real valued numbers between 0 and 1 . They are called listen preference and talk preference, respectively. These probabilities are used in the control loop of the agent - steps 'listen to others?' and 'talk to others?' in figure 1. In these steps, a random real is drawn and if the resultant value is under the (talk or listen) preference, the agent listens or talks to others.

\section{Evolution}

Agents underwent an evolutionary process of selection and variation. Agents with a high fitness were selected for and a variation of agents was accomplished by crossover of agent genotypes. The agent genes involved are the talk and listen preferences and the initial amount of sugar. Crossover happens by reproduction of two agents; this is not subject to individual decisions, nor is there any mate selection. If (1) two agents are on the same location or next to each other, (2) the genders differ, (3) their sugar levels are above the reproduction threshold, (4) they are both in the fertile age range, and (5) there is a vacant cell in the vicinity for placing the offspring, agents will always mate and generate offspring.

Reproduction takes place by crossover applied to two parents yielding the child, followed by a mutation operator on the child. The value of a gene in a child is the inherited value (from the wealthiest parent) plus a random number drawn from a the achievement of a centralized communications network may also entail other important costs not reflected in the current model. A messageboard, for example, would generally require more expensive means for carrying communications, such as more telephone links. These costs would also have to be included in judging the relative merits of various communications strategies.

Future work in this area should help to shed light on such issues. The results presented here represent the 'first returns' from a general testing platform that promises deeper insights. Ultimately, the authors hope that this general line of inquiry will help them discover the styles of communication that may be optimal for enabling the successful coordination and cooperation of agents in particular environments. Such insight will be crucial for engineering the cooperation-based function of collective robots and other decentralized technological systems.

Mark Buchanan 
Gaussian distribution with zero mean and fixed standard deviation. The child receives from each of the parents half of their sugar. The child inherits each of the values for vision, age of death, metabolism, and child bearing independently from one of the parents without change. After mating, each agent has a so-called recovery period, which is the number of cycles after mating that an agent cannot mate.

To illustrate the process of reproduction, consider the following example (without mutation). Two agents are next to each other: one with 24 sugar units, a listen preference of 0.7 and a talk preference of 0.55 ; the other has 16 sugar units, a listen preference of 0.6 and a talk preference of 0.5 . A child of these two agents gets its listen and talk preferences from the first agent $(0.7$ and 0.55 , respectively). Its initial sugar amount is the sum of half of the sugar amounts of each of the parents, thus 12 from the first parent and 8 from the second parent - its initial sugar amount is thus 20 .

\section{Experiments}

\subsection{Setup}

The experimental setup is shown in table 1. We varied four parameters:

- The cooperation threshold - in VUSCAPE this is implemented by the MSH (maximum amount of sugar that agent can harvest on its own). We varied the MSH from 0 to 10 in single increments.

- The number of resources - in VUSCAPE this is implemented by the MSS (amount of sugar that is distributed in the world). We varied the MSS from 1 to 10 in single increments.

- The communication protocol - this was either the centralized messageboard protocol or the decentralized newscast protocol.

- The communication parameters - these could be either fixed (agents always listen and talk) or evolutionary (agents learn to use their communication capabilities by evolution).

Table 1. An overview of the experimental setup

\begin{tabular}{|c|c|c|c|}
\hline \multirow{2}{*}{$\begin{array}{l}\text { maxSugarHarvest } \\
\text { (cooperation) }\end{array}$} & \multirow{2}{*}{$\begin{array}{l}\text { maxSugarSize } \\
\text { (resources) }\end{array}$} & \multicolumn{2}{|l|}{ Communication } \\
\hline & & protocol & parameters \\
\hline$[0 \ldots 10]$ & {$\left[\begin{array}{lll}1 & \ldots & 10\end{array}\right]$} & messageboard, newscast & evolutionary, fixed \\
\hline
\end{tabular}

Table 2. An overview of the experimental parameters

\begin{tabular}{|c|c|c|c|}
\hline \multirow{2}{*}{$\begin{array}{l}\text { Experiment } \\
\text { numberOfRuns }\end{array}$} & \multicolumn{2}{|c|}{ Agent } & \multirow[b]{2}{*}{$\exp$} \\
\hline & 10 & maxSugarHarvest (msh) & \\
\hline Scape & & singleStep & false \\
\hline height & 50 & initAgeZero & false \\
\hline width & 50 & minVision & 1 \\
\hline runLength & 2,000 & maxVision & 1 \\
\hline reseedSugar & true & minSugarMetabolism & 1 \\
\hline initialPopulation & 400 & maxSugarMetabolism & 1 \\
\hline sugarSeed.uniqueCell & false & minDeathAge & 60 \\
\hline sugarDistributionUnif & 3 & maxDeathAge & 100 \\
\hline sugarGrowBackRate & 1.0 & sexRecoveryPeriod & 0 \\
\hline numberOfSeeds & 1,000 & minReproductionSugar & 0 \\
\hline sugarDistributionType & uniform & allowSex & true \\
\hline maxSugarSize (mss) & $\exp$ & minlnitialSugar & 50 \\
\hline Cell & & maxInitialSugar & 100 \\
\hline allowMultipleAgents & true & preferNearestCell & false \\
\hline
\end{tabular}

Parameters indicated with 'exp' are varied in the experiments.

This makes a total of $11 \times 10 \times 2 \times$ $2=440$ experimental runs. Each run was done 50 times. An overview of all experimental parameter values is given in table 2 . Additionally, talk and listen features are inherited from the parent with the most sugar. The mutation sigma is 0.1 .

\subsection{Results}

The obtained results have been included in figures 2, 3 and 4 .

Figure 2 shows the results concerning the performance of the research populations ${ }^{2}$. Each data point corresponds to an environment with a certain need for cooperation. This need is expressed by the number of available resources (MSS) and the cooperation threshold (MSH). Each line should be interpreted as follows: in environments on and under the line, all runs resulted in extinction of the agent population. In environments above the line, the agent population size stabilized at some value. (Note that fig. 2 also contains the results of a benchmark experiment that we conducted without communication.)

Figures 3 and 4 show the results of the experimental runs where the communica-

\footnotetext{
2 This graph is a very dense presentation of many experimental runs. Each of the 5 graph lines [no communication, messageboard (fixed), newscast (fixed), messageboard (evolutionary) and newscast (evolutionary)] was extracted from the set of experimental runs covering the complete MSH $\times$ MSS range. These graphs can be found in Vink [15].
} 


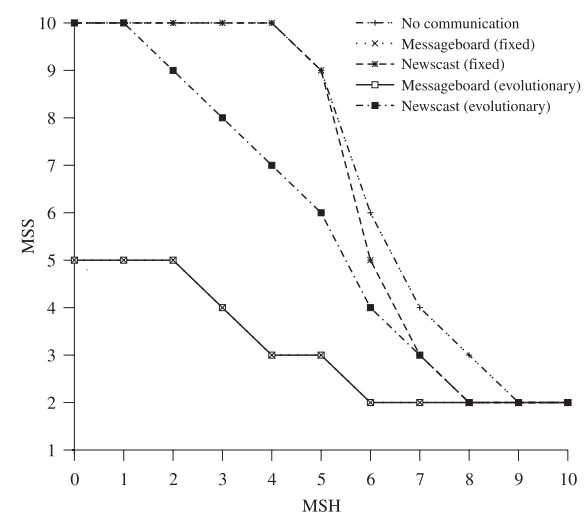

Fig. 2. Summary of the performance results of our comparison of protocols. Each data point corresponds to an environment with a certain need for cooperation. This need is expressed by the number of available resources (maximum sugarsize, mss) and the cooperation threshold (maximum sugar harvest, msh). Each line should be interpreted as follows: in environments on and under the line, all runs resulted in extinction of the agent population.

tion parameters were evolutionary. Each graph shows, for some given MSH and MSS, the final talk and listen preferences of these runs. In other words, every data point represents the average talk preference and average listen preference of all agents of the population at iteration 2000.

Because we used $10 \times 11=110$ different combinations of MSH and MSS, it is infeasible to give separate graphs for each environment; we therefore defined low $[\mathrm{MSH} \in(0-2)]$, medium $[\mathrm{MSH} \in(3-5)]$ and high $[\mathrm{MSH} \in(6-10)]$ cooperation thresholds. The ranges have not been chosen arbitrarily, but are related to the percentage of seeds that can be eaten by the agents when they are alone on a cell. They are chosen so that when the cooperation threshold is low, and when most sugar is available (MSS $=10$ ), the agents can harvest less than $25 \%$ of the seeds without the help of other agents. When the cooperation threshold is medium, the agents can harvest only about $50 \%$ of the seeds without the help of other agents. When the cooperation threshold is high, the agents can harvest nearly all seeds without help. Note
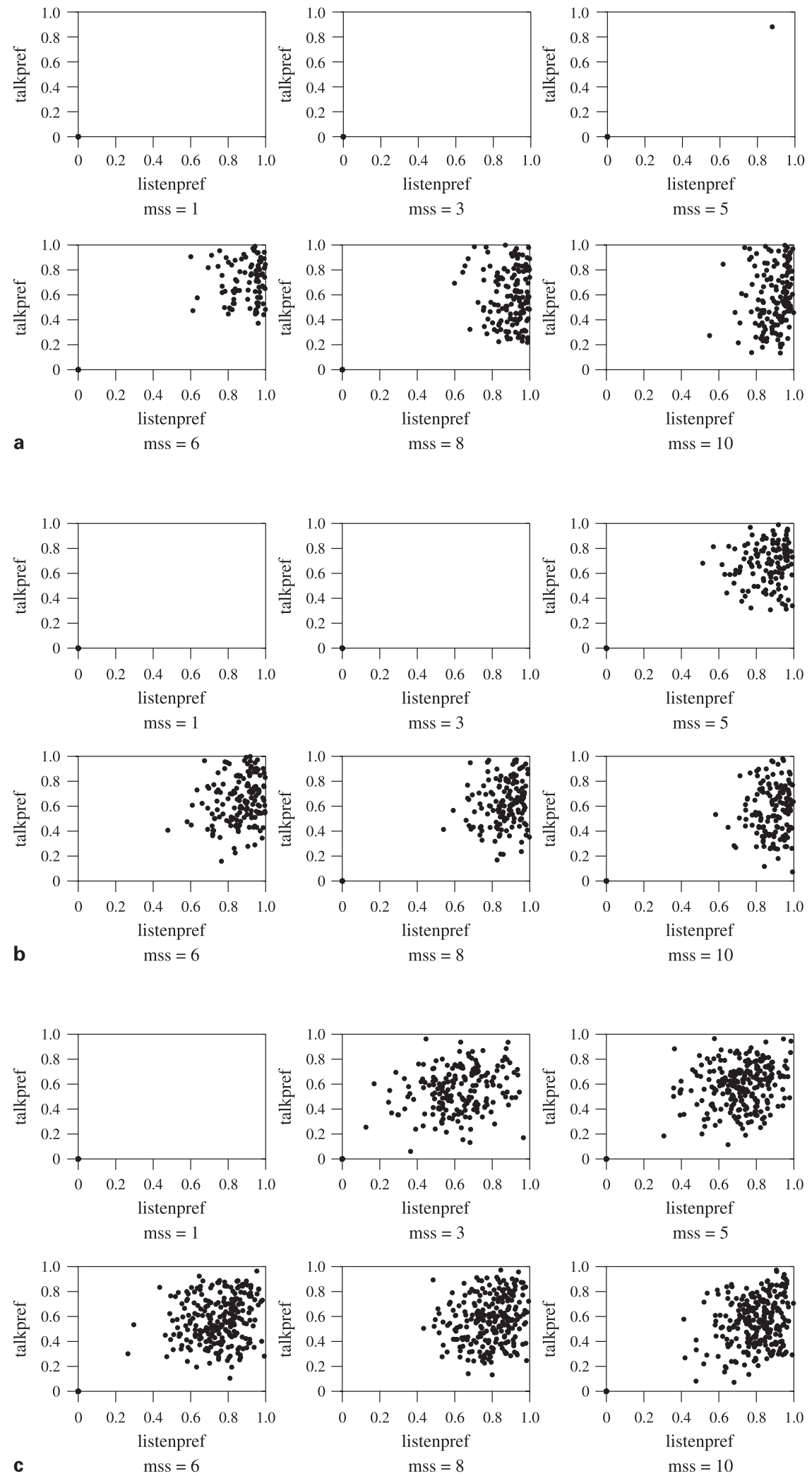

Fig. 3. Messageboard results (mss = maxSugarSize). a maxSugarHarvest $\in(0-2)$. b maxSugarHarvest $\in(3-5)$. c maxSugarHarvest $\in(6-10)$. 

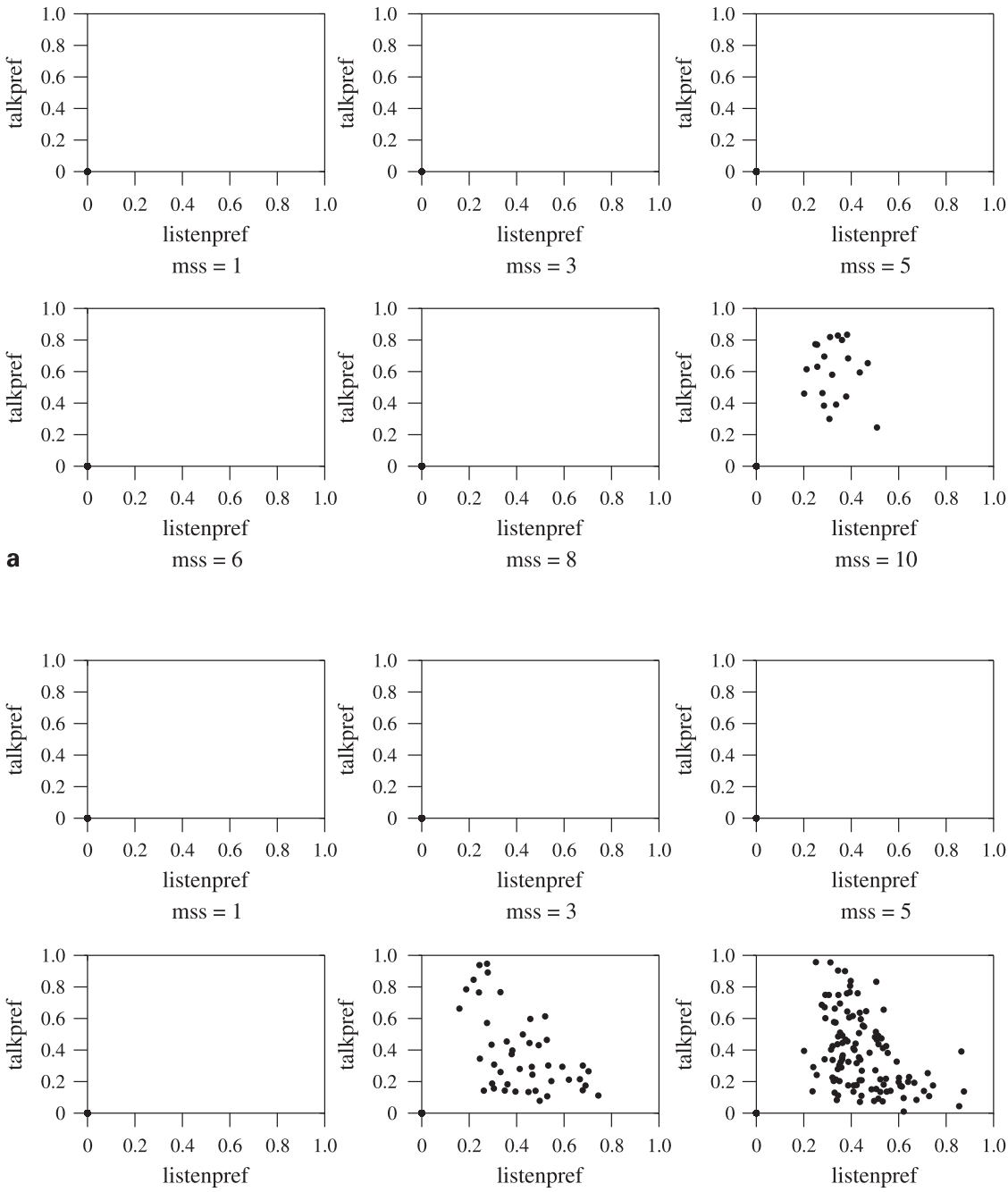

b
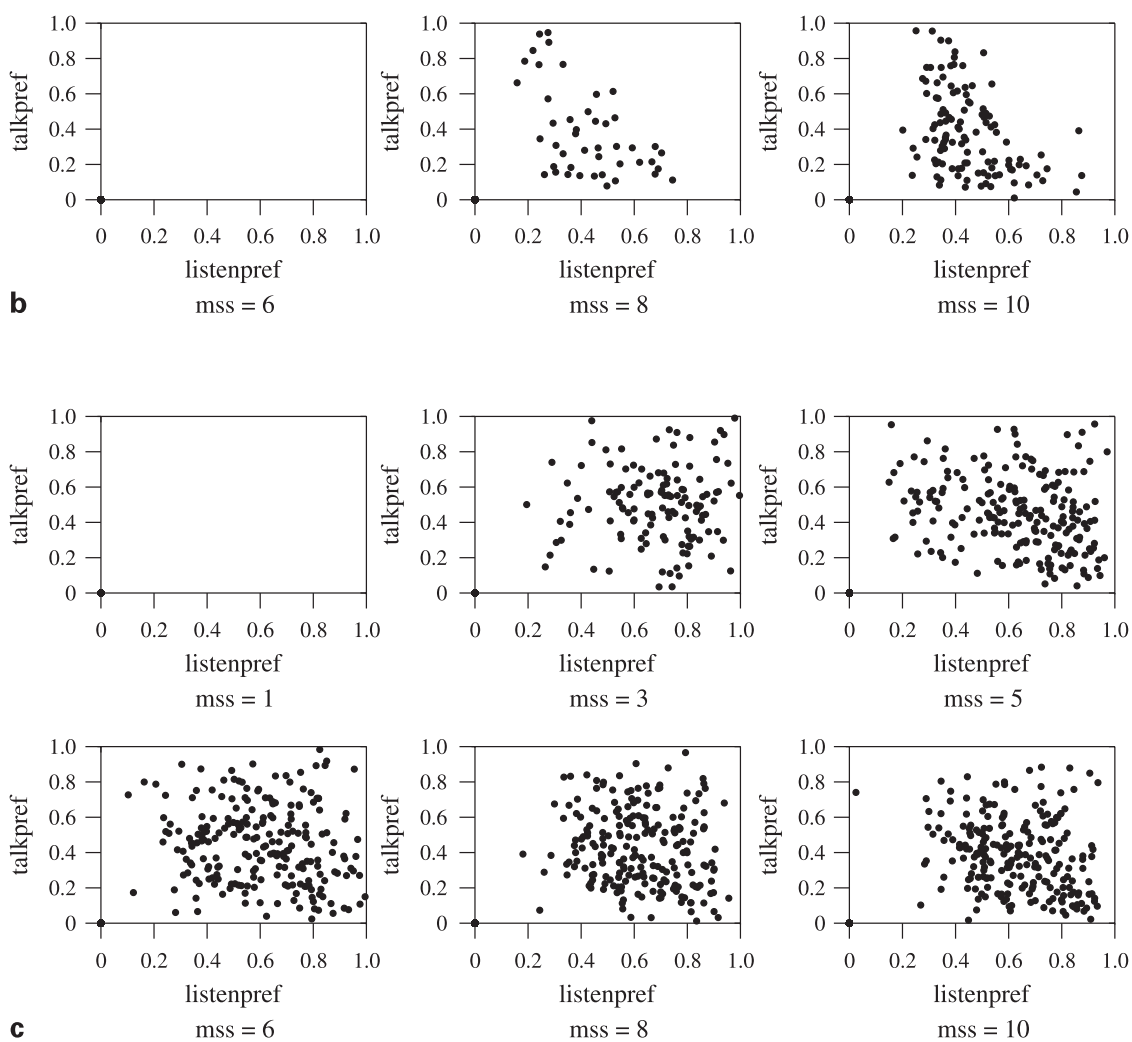

Fig. 4. Newscast results (mss = maxSugarSize). $\mathbf{a}$ maxSugarHarvest $\in(0-2)$. b maxSugarHarvest $\in(3-5)$. c maxSugarHarvest $\in(6-10)$. that the above-mentioned percentages are not stable during the simulation; they only apply to the initial situation where all seeds are at their maximum size. We did not include the graphs for mss $=2,4,7$, and 9; these can be found in [15].

\section{Analysis}

We analyze the results according to how we presented them in the graphs. Firstly, we analyze figure 2 that says something about the performance of the populations. Secondly, we analyze figures 3 and 4 to find out about the evolution of communication (what values did the talk and listen preferences evolve to?) in our experiments.

\subsection{Performance}

Figure 2 shows the performances of the populations $s^{3}$ under the investigated communication protocols. Each line in the graph represents an extinction border and should be read as such: on all data points under and on the graph line the population died out during the runs; on all environments above the line, the population stabilized at some value.

From the data from which we generated figure 2 (covering the complete MSH $\times$ MSS spectrum), we drew the following conclusions. (Note that these conclusions can only in part be observed in fig. 2; we included them here because of their relevance to the findings we make later.) We found that overall the messageboard protocol with fixed communication parameters performs much better than the newscast protocol with fixed communication parameters. Also for the evolutionary variant, we saw that the messageboard protocol outperforms the newscast protocol. However, the difference is smaller here, because the performance of the newscast

\footnotetext{
${ }^{3}$ Our notion of performance only takes into account the population size. In Vink [15] we present a more elaborate welfare function, but extensive testing demonstrated no significant difference between the population size and the population welfare for the environments that we investigate here. Hence, this paper only reports on the population sizes.
} 
protocol with evolutionary parameters performs better than with fixed parameters. In general, we saw that the messageboard performs better in the fixed variant than in the evolutionary variant, whereas the newscast protocol performs better in the evolutionary variant than in the fixed variant.

In figure 2 the graphs show that the messageboard (with evolution) performs best, followed by the newscast protocol with evolution, then by pure newscast and finally no communication, which performs worst. In more detail:

- Communication gives an advantage to the agents that use it. In figure 2 we see that communication improves the life expectancy of agents. All protocol variants we used extend the set of environments in which agent populations are viable. There are no environments in which agents using communication go extinct while agents which do not use communication survive; the set of environments in which agents not using communication survive is a true subset of the set of environments in which agents using communication survive.

- In environments in which agents can survive without using communication, the added value of communication is relatively small. When we only look at environments in which non-communicating societies survive, we see that adding communication leads to a relatively small increase in the 'performance' of the agent population. We contemplate that there exists some minimum population size for the population to survive; this may logically follow from the fact that the probability of reproduction depends on the population size. If the population size is too small, the probability of finding a partner for reproduction may also be too small. Based on our results, we expect the minimum population size necessary for survival to be somewhere close to 500 .

\subsection{Evolution of Communication} Messageboard (figure 3)

- Talk preference evolves to higher values when the need for cooperation increases. In figure 3 a we see that when the need for $\mathrm{Co}^{-}$ operation is high (mss $\leq 6)$, the runs in which the agent population survive result in higher average talk preference than when the need for cooperation is lower ${ }^{4}$ (e.g. mss $=10$ ). We observe the same effect in figure $3 b$. The effect is not visible in figure $3 c$; this is possibly because the selective pressure on higher talk preference is insignificant when the cooperation threshold is too high.

- Listen preference evolves to higher values when the need for cooperation increases. What we observed for talk preference also holds for listen preference. When looking at figure $3 c$ (high cooperation threshold) we see that as the value for mss increases, the surviving runs result in higher average listen preferences. The effect is also visible when comparing the corresponding graphs in figures $3 \mathrm{a}-\mathrm{c}$ to each other; when the cooperation threshold is low, the surviving runs result in higher average listen preferences than when the cooperation threshold is higher.

- Listen preference evolves to higher values than talk preference. In almost all graphs in figure 3 we see that listen preference evolves to higher values than talk preference. In other words, in almost all graphs we see that most points are below the diagonal from $(0$, $0)$ to $(1,1)$.

\section{Newscast (figure 4)}

- Talk preference does not evolve consistently. We observe no effects for the evolution of average talk preference in the results of the newscast protocol. The resulting average talk preferences of the surviv-

\footnotetext{
${ }^{4}$ We assume that the need for cooperation is lower when more sugar is available. Although more sugar also means larger seeds, and thus less seeds that can be harvested by single agents, we assume that the need for cooperation in order to survive is lower, because when agents harvest, they harvest more sugar.
}

ing agent populations seem to span the entire possible range for average talk preference. The evolution of talk preference observed may be due to genetic drift. Such genetic drift may explain some observation made in figure $4 \mathrm{c}$. In the bottom right corners of the graphs in this figure, we see many experiments in which nearly all agents talk whenever possible, while hardly any agents listen. We can also observe such results for the messageboard protocol, but they are much more prevalent in the results for the newscast protocol.

- Listen preference evolves to higher values than talk preference. As for the messageboard protocol, in the results of the newscast protocol we see that listen preference generally evolves to higher values than talk preference. Still, the effect is weaker than in the results of the messageboard protocol because of the earlier mentioned findings.

\section{Summary}

In both experiments, we observed that the listen preference evolves consistently to higher values than talk preference. This indicates that, for some reason, listening is more 'important' to the agents than talking. Although we think that this is because of the induced distribution of talk and listen events, we do not speculate further about what may be the reason - this is subject to future study.

\section{Conclusions}

The building blocks of a complex system with active entities are communication and cooperation. Communication is used for information exchange between the entities and cooperation is necessary if the entities want to achieve goals that are beyond their own reach. If we are to design complex artificial systems, we have to know the effects of deciding for available ways to communicate with respect to a given problem environment. This paper presented a first step towards such design questions. 
In a straightforward artificial society, we compared a centralized communication protocol (messageboard) with a decentralized protocol (newscast). The environments we investigated were scalable as we varied the need for cooperation and the available resources. In addition we considered hardwired agents (that always communicate) with learning agents (that evolutionary learn to communicate).

The results show that the performance of the messageboard protocol is much better than that of the newscast protocol in the environments that we examined. We observe this with both types of agents. Preliminary further investigation of this result indicates that the ratio between the speed at which information is distributed and the speed at which information loses its value is essential for the success of a given communication protocol [9]. Surprising still, with the newscast protocol, the learning agents outperformed the hardwired agents. Also, with the learning agents, we consistently observe that agents develop a higher preference for listening (receiving information from others) than talking (communicating information to others).

In the long run, we aim to design Emergent Collective Intelligence (ECI). The end goal of ECI research is to combine and exceed achievements in multi-agent systems [1], swarm intelligence [3], and evolutionary computation [10] research via developing synthetic methodologies such that groups of computationally complex agents produce desired emergent collective behaviours resulting from the bottom-up development of certain individual properties and social interactions. Forthcoming results will be published on different scientific forums; for locating them conveniently one can visit: http://www.cs.vu.nl/ci/eci.

\section{References}

1 Avouris N, Gasser L: Distributed Artificial Intelligence: Theory and Praxis. Dordrecht, Kluwer Academic Publishers, 1992.

2 Axelrod R: The Complexity of Cooperation: AgentBased Models of Competition and Collaboration. Princeton, Princeton University Press, 1997.

3 Bonabeau E, Dorigo M, Theraulaz G: Swarm Intelligence: from Natural to Artificial Systems. Oxford, Oxford University Press, 1998.

4 Buresch T: Evolutionary versus Lifetime Learning of Agents Controllers on a Gridworld. Technical Report. Amsterdam, Department of Computer Science, Vrije Universiteit, 2004. Available online at http://www.cs.vu.nl/ci/eci/.

5 Buzing PC, Eiben AE, Schut MC: Evolving agent societies with VUSCAPE; in Banzhaf W, Christaller T, Dittrich P, Kim JT, Ziegler J (eds): Advances in Artificial Life. Proceedings of the 7th European Conference on Artificial Life (ECAL 2003), Lecture Notes in Artificial Intelligence, Berlin, Springer, 2003, vol 2801, pp 434-441.

6 Collier N: Repast: an Extensible Framework for Agent Simulation. Technical Report. Chicago, Social Science Research Computing, University of Chicago, 2000.

7 Daniels M: Integrating simulation technologies with swarm; in Proceedings of the Workshop on Agent Simulation: Applications, Models, and Tools. Chicago, University of Chicago, 1999.

8 Eiben AE, Nitschke G, Schut MC: Comparison of reproduction schemes in an artificial society for cooperative gathering; in Proceedings of the AISB Socially Inspired Computing - Engineering with Social Metaphors Symposium. Technical Report. Hatfield, University of Hertfordshire, 2004.

9 Eiben AE, Schut MC, Toma T: Comparing multicast and newscast communication in evolving agent societies; in Beyer H-G, et al (eds): Proceedings of the Genetic and Evolutionary Computation Conference (GECCO 2005). New York, ACM Press, 2005, pp 75-82.

10 Eiben AE, Smith JE: Introduction to Evolutionary Computing. Berlin, Springer, 2003.

11 Epstein J, Axtell R: Growing Artificial Societies. Washington, Brookings Institute Press, 1996.

12 Jelasity M, Van Steen M: Large-Scale Newscast Computing on the Internet. Technical Report IR-503. Amsterdam, Department of Computer Science, Vrije Universiteit, 2002.

13 Parker M: Ascape: Abstracting Complexity. Technical Report. Washington, Center on Social and Economic Dynamics, The Brookings Institution, 2000.

14 Toma T: Communication in Artificial Societies Effects of Different Communication Protocols in an Artificial Environment. Technical Report. Amsterdam, Department of Computer Science, Vrije Universiteit, 2003. Available online at http://www.cs.vu.nl/ci/eci/.

15 Vink N: Exploring Communication Dynamics in VUSCAPE. Technical Report. Amsterdam, Department of Computer Science, Vrije Universiteit, 2004. Available online at http://www.cs.vu.nl/ci/eci/. 